\title{
Geometrization Theorem
}

Thurston's Geometrization Conjecture (now, a theorem of Perelman) aims to answer the question:

\section{How could you describe possible shapes of our universe?}

Here we will be assuming (although, some people think otherwise) that our space is 3dimensional (it has three directions: width, depth and height), compact (you cannot travel infinitely far away from a given point in space), closed (does not have a boundary), connected (you can get from any point to any point) and orientable (if you travel along a path and return to the point of departure, your left and right hand will not get switched).

In order to understand the answer to this question, we first look at the 2dimensional case for guidance. If our universe were a 2-d surface $S$, we could describe its shape by combining copies of three basic building blocks:

-The disk $D^{2} \quad$ (given by the inequality $x^{2}+y^{2} \leq 1 \quad$ ).

-The annulus $A^{2}$, also known as the cylinder (disk with one hole).

- The pair of pants $P^{2}$ (disk with two holes).

Each surface $S$ is obtained by gluing these blocks along their boundary circles. For instance, the 2-d sphere $S^{2}$ (given by the equation $x^{2}+y^{2}+z^{2}=1$ ) can be obtained by gluing together two disks (lower and upper hemispheres in $S^{2}$ ). The 2-d torus $T^{2}$ (the surface of a doughnut) is obtained by gluing together boundary circles of the cylinder (Fig. 1). ${ }^{1}$

Every surface $S$ can also be obtained as a connected sum of tori: Take a collection $T_{1}, \ldots, T_{g}$ of 2-d tori and attach them to the 2-sphere $S^{2}$ by first removing a disk from each torus and $g$ disks from $S^{2}$ and then identifying the boundary circles. Both descriptions of surfaces will be useful while dealing with 3-dimensional universes, which mathematicians call 3-d manifolds.

How can these descriptions of 2-d surfaces be generalized to dimension 3 ? We start with the obvious: Let's promote the 2-d building blocks to 3-dimensional ones by adding an extra spacial dimension (a circle) to each. For instance, we obtain the solid torus, $D^{2} \times S^{1}$, by rotating the 2-d disk around an axis in 3-d space which is far enough from the disk. The result is the 3-d doughnut. Similarly, one obtains $A^{2} \times S^{1}$ (a doughnut with a thinner concentric doughnut removed) and $P^{2} \times S^{1}$ (a doughnut from which we removed two thinner doughnuts). Note that the boundary of each 3-d block we obtained

1 Note that in our description of shapes we are not concerned with the measurements of distance: For instance, instead of the disk of radius 1 we could have taken, say, an ellipse. Mathematicians define this field of study as topology. 
consists of 1, 2 or 3 tori. Now, take a collection of these 3 -d blocks and glue them together along their boundary tori. The resulting spaces are called graph-manifolds. For instance, take the 3-d sphere $S^{3}$ given by the equation $x^{2}+y^{2}+z^{2}+w^{2}=1$ in 4-d space. One can describe $S^{3}$ as a graph-manifold by gluing together two solid tori: Take a small spherical cap on $S^{3}$ around the north pole $(0,0,0,1)$, then rotate this cap around the $x y$ plane. The result of the rotation is a solid torus. Removing this solid torus from $S^{3}$ leaves us with another solid torus. As a additional example, take the 3-d torus $T^{3}=S^{1} \times S^{1} \times S^{1} \quad$ : It can be obtained by gluing together the two boundary tori of $A^{2} \times S^{1}$.

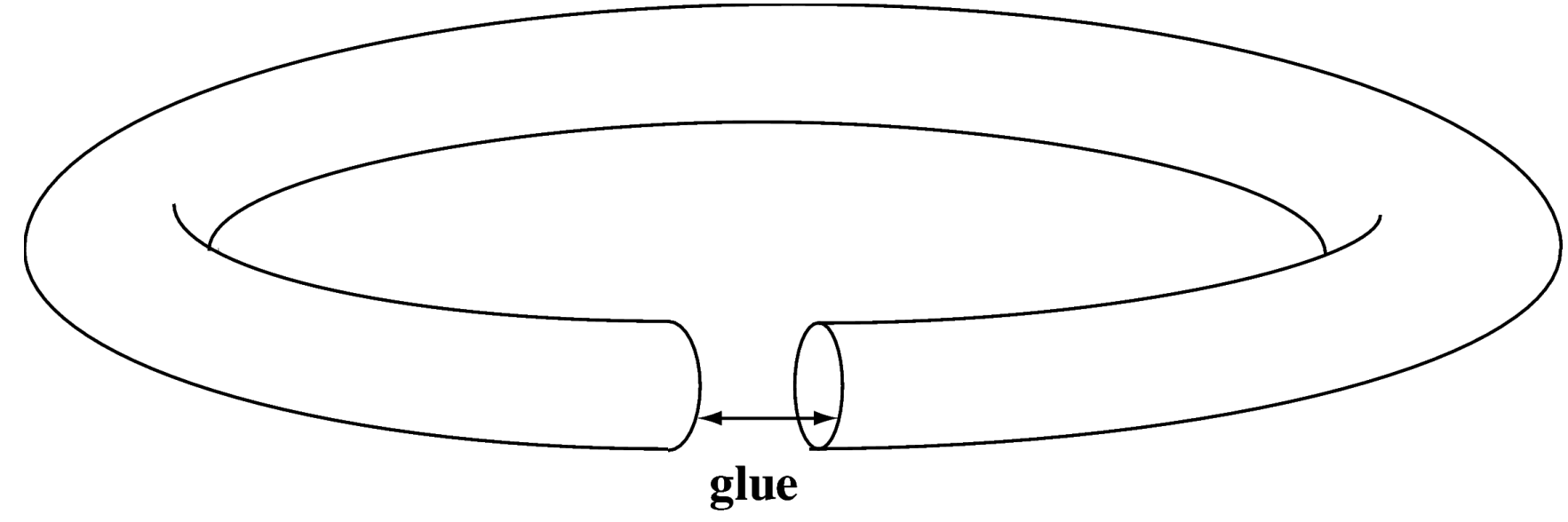

Fig. 1. Making a 2-d torus.

By analogy with the description of 2-d surfaces above, one could have expected that all 3-d manifolds are graph-manifolds. It turns out that this is far from being the case. The missing manifolds are best described by means of:

Hyperbolic geometry. On a small scale (say, the scale of a single planet), our universe appears flat: The distances between points in space can be computed by the Pythagorean formula, or, as mathematicians would say, by means of the Euclidean (flat Riemannian) metric which, in coordinates, is given by $d s^{2}=(d x)^{2}+(d y)^{2}+(d z)^{2}$. However, on the scale of a planetary system (and beyond), our universe is curved by gravity. The distance measurements are described by means of a Riemannian metric. To define such a metric we will use from now on the coordinates $x_{1}, x_{2}, x_{3}$; the notation $x$ will be reserved for a point in a manifold. Then a Riemannian metric is given by a metric tensor $g_{i j}$, so that $d s^{2}=\sum_{i, j} g_{i j} d x_{i} d x_{j}$. This metric tensor is required to be symmetric, i.e., $g_{i j}=g_{j i}$ and positive-definite, i.e., $d s^{2}$ is positive.

Hyperbolic 3-space $\mathbf{H}^{3}$ can be described as the upper half-space $\left(x_{3}>0\right)$ in 3-d space, with the Riemannian metric $d s^{2}=\frac{d x_{1}^{2}+d x_{2}^{2}+d x_{3}^{2}}{x_{3}^{2}}$. In this description, hyperbolic space does not seem to have any preferred origin, but appears to have a preferred direction. To dispel this misperception, let's change the coordinates and describe the 
hyperbolic metric on the unit ball $x_{1}^{2}+x_{2}^{2}+x_{3}^{2}<1: \quad d s^{2}=4 \frac{d x_{1}^{2}+d x_{2}^{2}+d x_{3}^{2}}{1-\left(x_{1}^{2}+x_{2}^{2}+x_{3}^{2}\right)^{2}}$. It is then clear that such a metric is homogeneous (it looks the same at each point) and isotropic (is the same in every direction). Mathematicians describe this property by saying that the hyperbolic metric has constant curvature. There are three classes of constant curvature metrics: Of positive curvature (the metric on the sphere), zero curvature (the metric of flat space) and negative curvature (the hyperbolic metric). In order to visualize what negative curvature means, we note that the volume enclosed by a sphere of (hyperbolic) radius $r$ in hyperbolic space grows exponentially fast for large $r$. (In flat 3-d space the volume grows only cubically.)

How can our universe possibly have negative curvature? At first glance, this would violate our assumption that it is closed (compact and borderless). Let's reexamine the flat metric. Start with a cube $Q$ in the flat 3-d space. Take opposite faces of $Q$ and identify them by parallel translations. The result has the shape of the 3-d torus $T^{3}$ which then inherits a flat Riemannian metric from $Q$ since the translations are isometries (they preserve the metric).

How does this help to construct closed hyperbolic manifolds? Imagine that you have a finite polyhedral convex solid $P$ in $\mathbf{H}^{3}$ and a collection of isometries of $\mathbf{H}^{3}$ identifying the faces of $P$. The result of identification (subject to certain conditions) is a compact hyperbolic 3-d manifold $M^{3}$. Its Riemannian metric is the one inherited from $P$. One of the earliest examples of this construction is the Seifert-Weber dodecahedral space, obtained from a right-angled hyperbolic dodecahedron $P$ (Fig. 2).

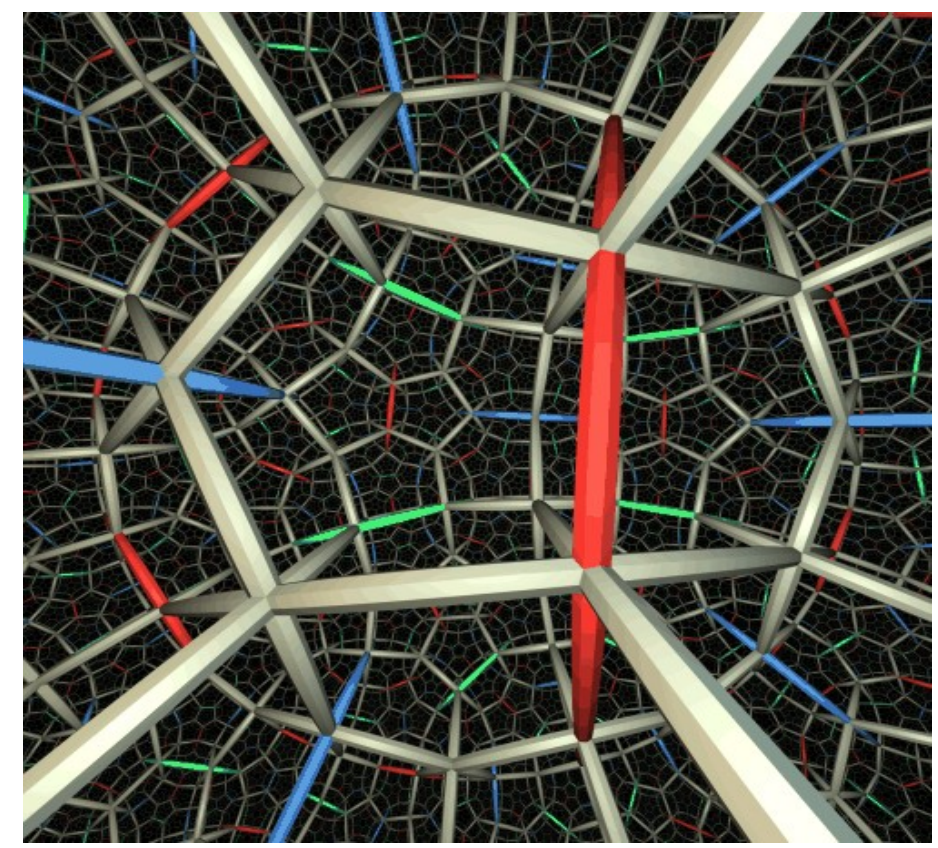

Fig. 2. Life in Seifert-Weber dodecahedral space (courtesy of Geometry Center, 
University of Minnesota).

More generally, one can take a convex finite volume solid $P$ in $\mathbf{H}^{3}$ with finitely many faces ( $P$ could have infinite diameter, see Fig. 3), to obtain a hyperbolic 3-d manifold $M^{3}$ of finite volume. The (ideal) boundary of such an $M^{3}$ would be a collection of 2-d tori. These tori $T^{2}$ satisfy an important technical property, they are incompressible in $M^{3}$ : If a circle on $T^{2}$ bounds a 2-d disk in $M^{3}$, then it already bounds a disk in $T^{2}$. The resulting manifolds $M^{3}$ are hyperbolic and every finite volume hyperbolic manifold appears this way. It is common to say that a 3-d manifold (with unspecified geometry) is hyperbolic if it admits a hyperbolic metric of finite volume (such metric is known to be unique).

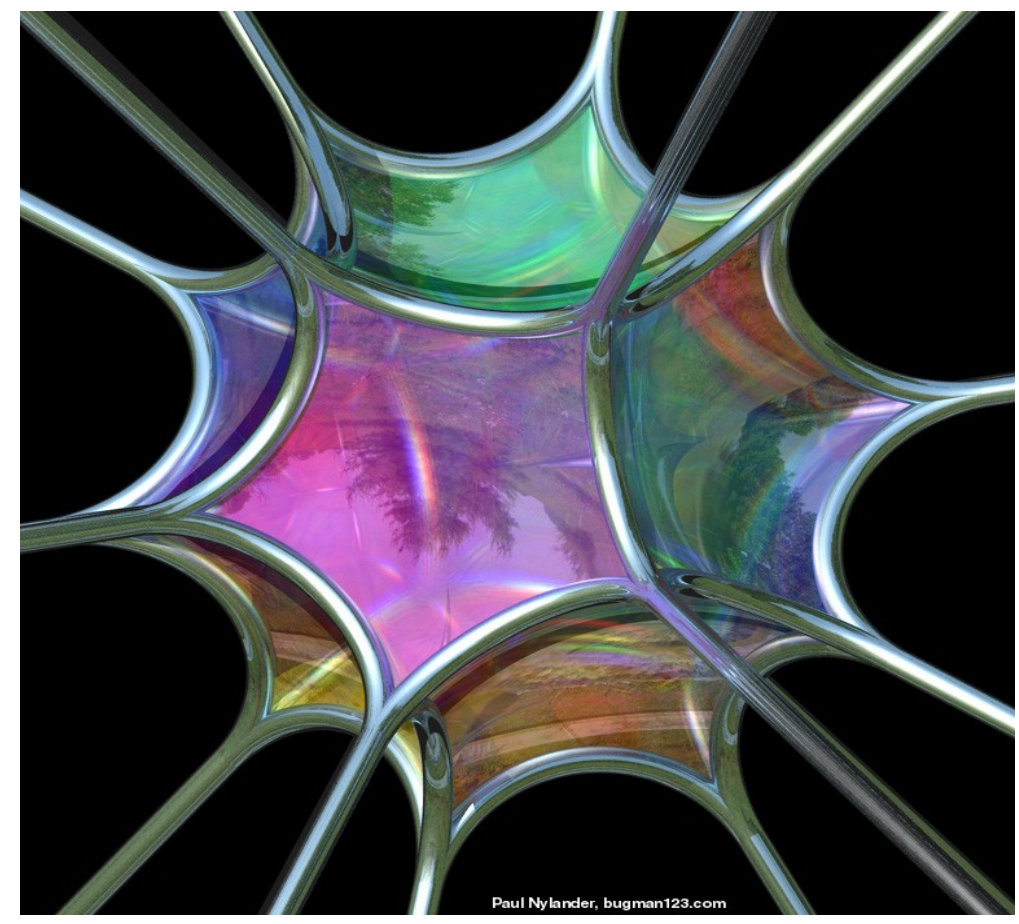

Fig. 3. Hyperbolic dodecahedron of infinite diameter. Courtesy of

Paul Nylander, http://nylander.wordpress.com/2007/02/15/hyperbolic-dodecahedron

One can show that hyperbolic manifolds are never graph-manifolds, so we indeed have a new class of possible universes. More generally, a manifold is called geometric if it admits a Riemannian metric which is locally homogeneous (i.e., it locally looks the same at all points). Besides the constant curvature metrics, there are 5 more homogeneous metrics: They are (twisted) products of 1-d and 2-d homogeneous metrics (for instance, $\mathbf{S}^{2} \times \mathbf{R}$ and $\mathbf{H}^{2} \times \mathbf{R}$ ); see [4]. We are now ready to state 
Thurston's Geometrization Conjecture (TGC). Every (closed, oriented) 3-d manifold $M^{3}$ can be obtained as follows: Start with a finite collection of graphmanifolds (with incompressible boundary if there is any) and finite volume hyperbolic manifolds. Then glue these manifolds along their respective boundary tori so that the result has no boundary. It is possible that the resulting manifold $M^{\prime}$ is disconnected (you cannot reach one point from another). To remedy this, take a connected sum of the components of $M^{\prime}$ : Remove small balls from distinct components and glue together the exposed boundary $2-\mathrm{d}$ spheres. The result is $M^{3}$.

In other words, one can build $M^{3}$ by using the three types of basic 3-d building blocks appearing in graph-manifolds, together with hyperbolic manifolds. Equivalently, one can state TGC by saying that $M^{3}$ can be built from a collection of geometric manifolds by gluing them along incompressible boundary tori and then taking a connected sum.

William Thurston proved his conjecture in the 1970s under the technical assumption that $M^{3}$ is Haken. This covered a large class of 3-d manifolds but far from all of them. For his work, Thurston was awarded a Fields Medal in 1983. (The Fields Medal is one of the highest honors a mathematician can receive, awarded to mathematicians below the age of 41 ; it is awarded once every 4 years to 2-4 mathematicians.) An important special case of TGC is the Poincaré Conjecture, to which Thurston's technique did not apply:

Poincaré Conjecture (PC): If $M^{3}$ is a closed simply-connected ${ }^{2} 3$-d manifold, then $M^{3}$ is the 3-d sphere.

This conjecture was first formulated by Henri Poincare in the early 20th century and, despite of numerous efforts, remained out of reach until Perelman's work. Its analogs were proven for higher-dimensional manifold (dimensions $\geq 5$ by the $1960 \mathrm{~s}$ and dimension 4, in the topological form, by the 1980s) through efforts of many mathematicians (most notably, S.Smale, J.Stallings and M.Freedman).

Another special case of TGC is

Spherical Space Forms Conjecture (SSFC): Suppose that $M^{3}$ is a closed 3-d manifold with finite fundamental group ${ }^{3}$. Then $M^{3}$ is a spherical space-form, i.e., it admits a metric of constant positive curvature. In particular, such $M^{3}$ is a graphmanifold.

2 I.e., every circle in $M^{3}$ can be contracted to a point.

3 In the context of 3-d manifolds, this property can be formulated as follows: If $c$ is any circle in the manifold $M^{3}$ then, for some $n>0$, the circle $c^{n}$ obtained by tracing the original circle $n$ times, can be contracted to a point in $M^{3}$. 
Ricci Flow. The Ricci Flow (RF) was introduced by Richard Hamilton as a possible approach to TGC. Hamilton used it to prove TGC for manifolds of positive curvature in 1982. He also wrote a number of other papers establishing a program for proving TGC using the RF.

Roughly speaking, the idea is to define a flow on the space of Riemannian metrics that, starting with an arbitrary metric tensor $g$ on a 3-d manifold, "homogenizes" $g$ and in the limit separates the manifold into graph-manifolds and homogeneous pieces. To define the RF, consider a metric tensor $g_{i j}(x)$ on a 3-d manifold $M$. This metric can be encoded in a 3-by-3 array of numbers, indexed by $i$ and $j$, that depend on the point $x$ in $M$. The curvature of $g$ is described by means of the Riemannian curvature tensor $R_{i p j q}$ (given by a 4-d array of numbers). This tensor has an important simplification, the Ricci tensor $\operatorname{Ric}(g)=\operatorname{Ric}_{i j}=\sum_{p, q} R_{i p j q} g^{p q}$ which is a symmetric ( $\left.R i c_{i j}=R i c_{j i}\right)$ 2-d array of numbers. Then, the RF is given by the differential equation $\frac{\partial g(t)}{\partial t}=-2 \operatorname{Ric}(g(t))$. Ricci flow can be regarded as an analogue of the heat flow, describing the evolution of temperature in flat space: As the heat flow homogenizes the temperature distribution in space, the RF homogenizes Riemannian metrics.

By rescaling both space and time, one gets Normalized Ricci Flow (NRF) of metrics of constant volume: $\hat{g}^{\prime}(t)=-2 \operatorname{Ric}(\hat{g}(t))+\frac{2}{3} r \hat{g}(t)$. Here $r=r(t)$ is some scalar function. Suppose that $\hat{g}\left(t_{0}\right)$ is a stationary point of the NRF, i.e., $\hat{g}^{\prime}\left(t_{0}\right)=0$. Then $\hat{g}\left(t_{0}\right)$ is Einstein: Its Ricci tensor is a scalar multiple of $\hat{g}\left(t_{0}\right)$. In dimension 3, Einstein metrics all have constant curvature, hence $M^{3}$ is geometric! This suggests the strategy: Start with an arbitrary metric $g(0)$ on $M^{3}$ and let it evolve via the NRF, hoping that it will converge to a stationary metric (that will have to be homogeneous). This strategy actually works if $g(0)$ has positive curvature (Hamilton, 1982). However, it was known since the early 1980s that in many cases, 2-d spheres inside of $M^{3}$ can cause the NRF to blow up (the curvature becoming infinite) in a finite amount of time.

Dumbbell Example: Take two copies of the round 3-d sphere and connect them by a thin neck. The neck will get pinched (under both RF and NRF) in finite time as the 2-d sphere in its cross-section gets pinched into a point (and has curvature blowing up to infinity in the process) as $t \rightarrow T_{1}<\infty$ and our "universe" splits in two (Fig. 4). 

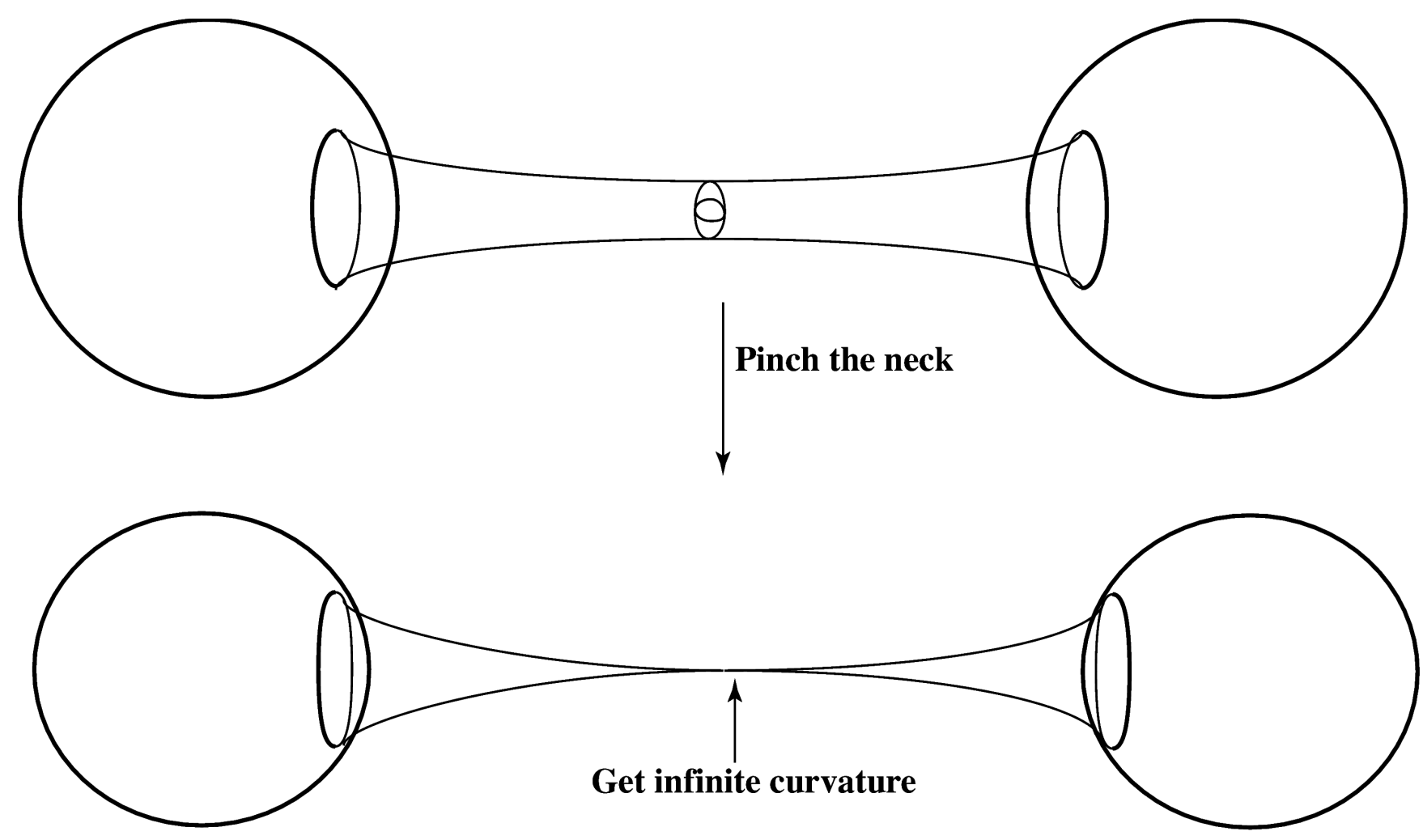

Fig. 4. Neck pinching.

In this example the RF "finds" the 2-d spheres along which $M^{3}$ has to decompose as a connected sum (before becoming geometric). It also suggests that one should look for these spheres in the part $M(t)^{+}$of $M^{3}$ where the curvature of $g(t)$ is "high." Hamilton was hoping that one can prove that the regions of high curvature on $M^{3}(t)$ (and the corresponding singularities at the first blow-up time) are "nearly standard," similar to the dumbbell example.

Understanding singularities of $g$ at the first blow-up time $T_{1}$ was the first among major obstacles on the way of Hamilton's program by the mid 1990s. This was when Gregory Perelman decided to work on the problem. Working in nearly total isolation and secrecy from the mid 1990s to 2002, Gregory Perelman introduced several new important tools and ideas and modified Hamilton's program; his work culminated in a sequence of three preprints in 2002-2003 proving the entire TGC. Instead of looking at the RF as just an evolution of a metric on a 3-d manifold $M^{3}$, he analyzed geometry of the 4-dimensional manifold $N^{4}$ obtained from $M^{3}$ by adding the extra dimension (the time $t$ ). This 4-dimensional geometry is specifically designed to reflect properties of the RF. Using this geometry (in combination with the Alexandrov geometry of singular metric spaces), Perelman established that singularities of the RF are, indeed, standard after appropriate rescaling and taking a limit. This allowed him to prove, without identifying the geometry of singularities precisely, that the topological type of high curvature regions $M(t)^{+}$in $M(t)$ is standard, the main examples being: A cup (a 
3-d ball), spherical neck (product of the 2-d sphere and the interval) or a spherical space-form (a 3-d manifold which admits a metric of positive curvature). On the other hand, Perelman managed to understand the geometry of $M(t)^{+}$well-enough to be able to cut it off from the rest of $M^{3}$ along some spheres and attach nearly round spherical caps along these spheres (Fig. 5).
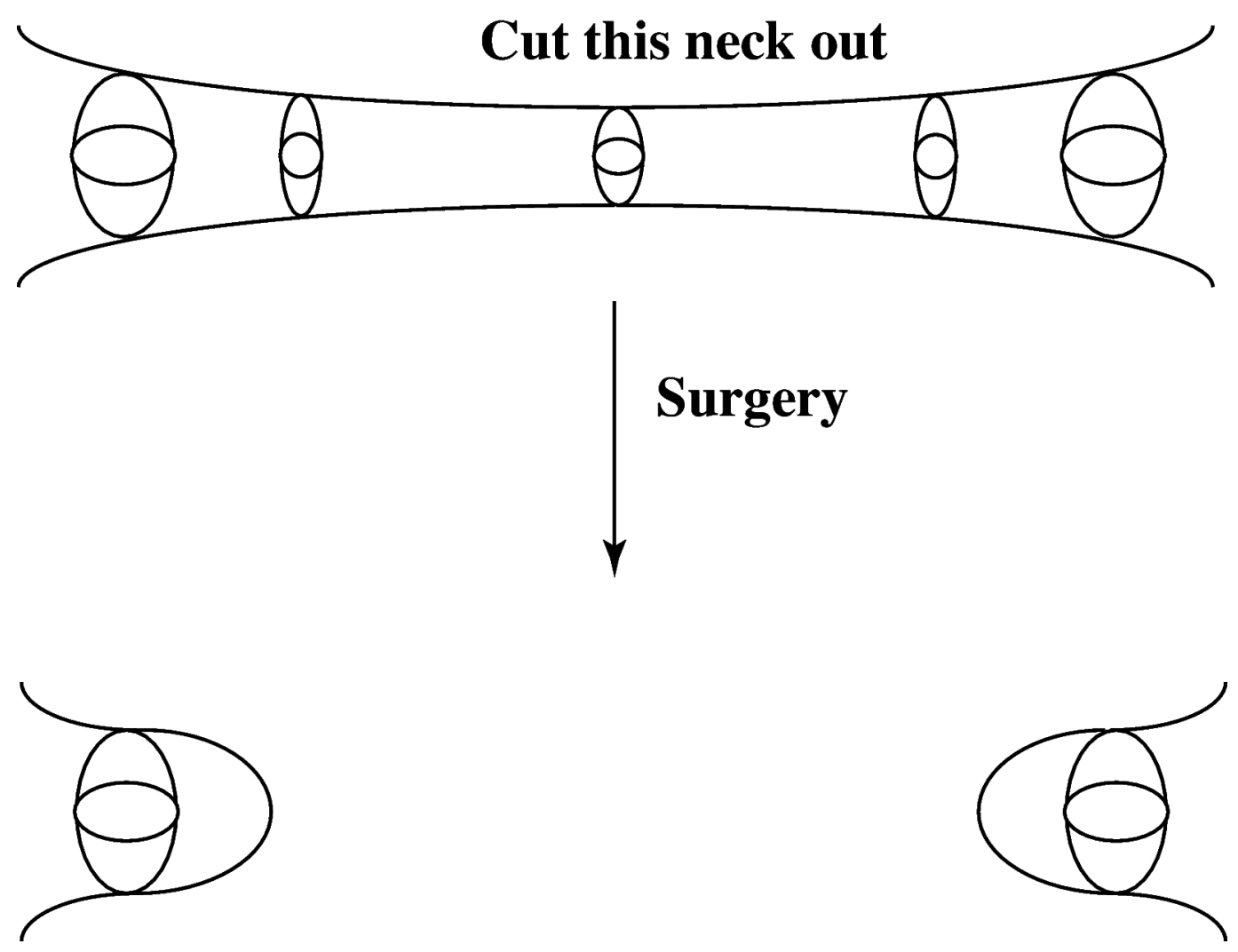

Fig. 5. Surgery.

As a result, Perelman has defined (realizing an earlier idea of Hamilton) the Ricci Flow with Surgeries (RFWS) which evolves both the geometry and the topology of $M^{3}$. He proved that the surgery times $T_{i}$ are well-separated and the RFWS thus exists for all times $t \geq 0$. Perelman also proved that (under certain assumptions) the entire manifold becomes extinct in a finite time, the curvature becoming infinitely high everywhere. (This was also independently established by Colding and Minnicozzi.) This means that the original manifold was the connected sum of spherical space-forms. In particular, this happens if $M^{3}$ is simply connected or, more generally, has finite fundamental group; this yields the Poincaré Conjecture and the Spherical Space Forms Conjecture. Lastly, extending prior work of Hamilton, Perelman proved that at $t=\infty$, the manifold $M(t)$ splits along incompressible tori into pieces $M_{\text {thick }}$ where the metric becomes hyperbolic and the pieces $M_{\text {thin }}$ where the metric collapses to metrics of 
dimension 1 or 2 .

This work was described by Perelman in three preprints that he posted on the internet in 2002-2003: "Entropy formula for the Ricci flow and its geometric applications," "Ricci flow with surgery on 3-manifolds," and "Finite extinction time for the solutions to the Ricci flow on certain three-manifolds." In order to establish the full TGC one also needs to show that $M_{\text {thin }}$ is a graph-manifold. A proof of the latter (originally promised by Perelman) was soon provided by T.Shioya and T.Yamaguchi, and, later on, by other mathematicians.

So far, Perelman's papers were not published in a peer-reviewed journal (and he does not seem to be interested in this), but only posted on the Mathematical Archive http://arxiv.org/, which is a common place for mathematicians to post preliminary versions of their work. Many arguments in Perelman's proofs are rather sketchy and some important details missing. Soon after the appearance of Perelman's preprints, several teams of mathematicians started to work towards filling in the missing details. Three detailed accounts of Perelman's proof of TGC have emerged:

1.As early as Summer of 2003, B.Kleiner and J.Lott started to post missing details of Perelman's proof on the internet; their work culminated in the article [2] covering the entire proof.

2.J.Morgan and G.Tian published a book [3] with a self-contained treatment of the PC (and SSFC) part of TGC.

3.H.-D.Cao and X.-P.Zhu's account of the entire proof published in [1] caused a great deal of controversy that spilled over to the popular press, including the New Yorker.

For his contributions to geometry, including the proof of TGC, Perelman was awarded the Fields Medal (that he declined to accept) at the International Congress of Mathematicians in Madrid in 2006.

Why do mathematicians care about TGC? The proof of TGC solved a number of important open problems (for instance, the PC); TGC also allows to reduce many open problems about 3-d manifolds to questions about hyperbolic manifolds, which then can be attacked using geometric, analytic or algebraic methods. For instance, one can define an algorithm, using TGC, which given two 3-dimensional manifolds as its input, will determine if they have the same topological shape or not. Such an algorithm is known to be impossible for manifolds of dimension $\geq 4$.

Why is TGC relevant outside of the mathematical community? TGC deals with possible shapes of our universe. Determining the actual shape of our space is an important problem in cosmology which is best understood in terms of TGC. For instance, astronomers, following the idea of J.-P.Luminet, have recently attempted to 
verify, using background radiation, if our universe has the shape of a certain space-form (Poincaré Dodecahedral Space).

Michael Kapovich

\section{Bibliography.}

[1] H.-D.Cao and X.-P.Zhu, A complete proof of the Poincaré and geometrization conjectures

- application of the Hamilton-Perelman theory of the Ricci flow, Asian J. Math., vol. 10 (2006), p. 165-492.

[2] B.Kleiner and J.Lott, Notes on Perelman's papers, Geometry \& Topology, vol. 12 (2008), p. 2587-2855.

[3] J.Morgan and G.Tian, Ricci flow and the Poincaré conjecture, vol. 3 of Clay Mathematics Monographs, American Mathematical Society, Providence, RI, 2007.

[4] W.Thurston, Three-Dimensional Geometry and Topology, I, vol. 35 of Princeton Mathematical Series, Princeton University Press, 1997.

\section{Supplementary References:}

G.Besson, The geometrization conjecture after R. Hamilton and G. Perelman, Rend. Semin. Mat. Univ. Politec. Torino, vol. 65 (2007), no. 4, p. 397-411.

T.Colding, W.Minicozzi II, Estimates for the extinction time for the Ricci flow on certain 3-manifolds and a question of Perelman, J. Amer. Math. Soc. vol. 18 (2005) 561569

M.Kapovich, Hyperbolic manifolds and discrete groups. Progress in Mathematics, vol. 183. Birkhäuser Boston, Inc., Boston, MA, 2001.

J.Morgan, Recent progress on the Poincaré conjecture and the classification of 3manifolds, Bulletin Amer. Math. Soc. vol. 42 (2005) no. 1, p. 57-78.

J.-P.Otal, The hyperbolization theorem for fibered 3-manifolds. SMF/AMS Texts and Monographs, vol. 7. American Mathematical Society, Providence, RI; Société Mathématique de France, Paris, 2001.

T.Shioya, T.Yamaguchi, Volume collapsed three-manifolds with a lower curvature bound, Math. Ann. vol. 333 (2005), no. 1, 131--155. 